\title{
MOLECULAR GAS IN GALACTIC NUCLEI
}

\author{
Nick Scoville \\ Owens Valley Radio Observatory \\ California Institute of Technology
}

\begin{abstract}
Recent high resolution interferometric observations of the molecular gas in luminous IR galaxies reveal extraordinary concentrations of star forming material in the central few kpc. In several of the nearest IR bright galaxies, the molecular gas in the central regions is concentrated in a bar-like distribution (IC342, NGC 6946, and NGC 253) and in NGC 1068 , approximately $40 \%$ of the molecular gas is confined to two arms or a ring at approximately $1.6 \mathrm{kpc}$ radius. Interferometry on the most luminous galaxies $\left(L_{I R} \geq 10^{11} L_{\odot}\right)$ reveals that approximately half of the total interstellar matter is contained in the central kpc with mean densities of several hundred $\mathrm{H}_{2} \mathrm{~cm}^{-3}$. Such gas concentrations should result in the very rapid formation of stars, i.e. a central star burst yielding a massive central star cluster.
\end{abstract}

\section{LUMINOUS GALAXIES}

In the nearby bright IR galaxies, high resolution millimeter-wave interferometry and single dish observations have revealed a variety of morpohologies in the neutral gas. Three of the galaxies first mapped with the Owens Valley millimeterwave inteferometer showed elongated bar-like distributions for the molecular gas in the central kpc. The results for IC 342 and NGC 6946 have been published by Lo et al. (1984) and Ball et al. (1985). The more recent CO interferometry for NGC 253 consisting of a mosaic of seven 1' fields (Canzian, Mundy, and Scoville 1988) shows a massive bar of molecular gas aligned with the stellar bar seen in optical and near infrared maps (Scoville et al. 1985).

A rather different morphology is found in the nearby Seyfert II galaxy NGC 1068. In this case, approximately $4 \times 10^{9} M_{\odot}$ of molecular gas resides in a ring at the outer edge of the bright optical disk (Myers and Scoville 1986). This ring of neutral gas situated just outside the stellar bar recently discovered in the near infrared (Scoville et al. 1988) is somewhat surprising in view of the abundant evidence for a high rate of star formation in the interior optical disk. On the other hand, the kinematics of the molecular gas indicate a substantial component of radial motion suggesting that at times in the past, there has been an abundance of star forming material within the central disk.

Perhaps most dramatic in terms of star burst activity are the high luminosity and ultraluminous galaxies discovered as a result of the IRAS survey. At the higher 


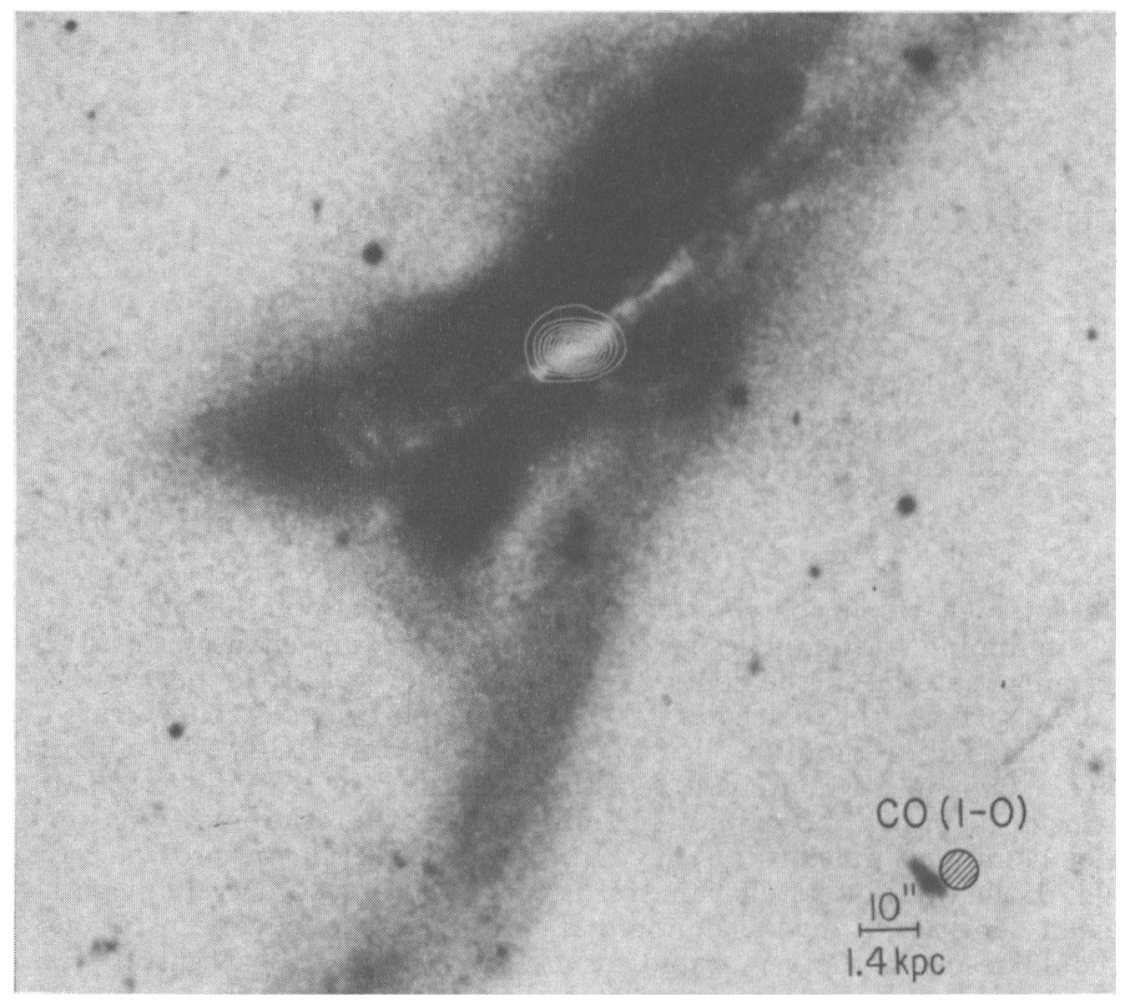

Figure 1: Integrated intensity map of $\mathrm{CO}$ emission in NGC 520 superimposed on the optical photograph from the Arp atlas. Contour levels are $10 \%$ of peak. The hatched beam symbol indicates both the position angle and size of the synthesized beam (Sanders et al. 1988).

luminosities, one sees a high preponderance of double nuclei and/or extended tidal tails indicative of strong galactic interactions or the merging of two galaxies. It is also evident that the optical spectra of the ultraluminous galaxies are dominated by non-thermal emission characteristic of a narrow line AGN or Seyfert nucleus rather than thermal HII region-type spectra seen in the lower luminosity galaxies. This qualitative assessment of the optical data strongly suggests that the highest luminosities are initiated by galactic collisions, and the dominant energy source may in fact be a non-thermal $A G N$. Virtually all the luminous IRAS galaxies have also been shown to be extremely rich in interstellar gas, predominantly molecular hydrogen (e.g. Sanders, Scoville, and Soifer 1987).

The molecular gas gas is also highly concentrated in their nuclei. Over the last two years, the millimeter wave interferometer at Owens Valley Radio Observatory has been used for aperture synthesis mapping of the $\mathrm{CO}$ emission in eight of the luminous galaxies (Scoville et al. 1986, Sargent et al. 1987, Sanders et al. 1988, Scoville et al. 1989). In each case, 30-70\% of the total CO emission is confined to a region $\leq 10^{\prime \prime}$ in size, centered on one of the galactic nuclei. Most 
spectacular is Arp $220\left(\mathrm{~L}_{I R}=1.3 \times 10^{12} \mathrm{~L}_{\odot}\right)$ where $1.3 \times 10^{10} \mathrm{M}_{\odot}$ of $\mathrm{H}_{2}$ is contained within the central $\mathrm{R} \leq 750 \mathrm{pc}$. The mean molecular gas density averaged over a spherical volume of this size is approximately $200 \mathrm{H}_{2} \mathrm{~cm}^{-3}$. The ratio of far infrared luminosity to $\mathrm{H}_{2}$ mass is $100 \mathrm{~L}_{\odot} / \mathrm{M}_{\odot}$. For comparison, the mean value of this luminosity-to-mass ratio in Galactic GMCs is $3 \mathrm{~L}_{\odot} / \mathrm{M}_{\odot}$ and the maximum value, obtained in localized areas immediately adjacent to galactic HII regions, is $40 \mathrm{~L}_{\odot} / \mathrm{M}_{\odot}$. Thus, in Arp 220 the overall "star formation" efficiency is 30 times the average in the Milky Way.

We have recently undertaken a theoretical investigation of the evolution of interacting gas-rich galaxies (Norman and Scoville 1988, Scoville and Norman 1988). Our model starts with the formation of a single coeval stellar cluster of total mass $4 \times 10^{9} \mathrm{M}_{\odot}$. This mass is distributed among stars with a Salpeter initial mass function $(\alpha=2.35)$ over the range $1-50 \mathrm{M}_{\odot}$. The total number of stars in the cluster is $1.4 \times 10^{9}$ and evolution of the stellar population is followed. The small radius of the cluster $(10-50 \mathrm{pc})$ implies an extremely high escape velocity $\left(>10^{3} \mathrm{~km} \mathrm{~s}^{-1}\right)$ ensuring that all mass-loss occurring during the late stellar evolution phases (red giant mass-loss and supernovae) will be trapped in the cluster and eventually sink, dissipatively, to the center. Thus, based on standard stellar evolution and an assumed initial mass function, it is possible to predict the growth rate of the central black hole and its accretion luminosity.

\section{REFERENCES}

Ball, R., Sargent, A.I., Scoville, N.Z., Lo, K.Y., and Scott, S.L. 1985, Ap.J. (Letters), 298, L21

Canzian, B.J., Mundy, L.G., and Scoville, N.Z. 1988, in preparation

Lo, K.Y. et al. 1984, Ap.J. (Letters), 282, L59

Myers, S.T. and Scoville, N.Z. 1986, Ap.J. (Letters), 312, L39

Norman, C.A. and Scoville, N..Z. 1988, Ap.J. 332, 163

Sanders, D.B., Scoville, N.Z., Sargent, A.I., and Soifer, B.T. 1988, Ap.J. (Letters), 324, L55

Sargent, A.I., Sanders, D.B., Scoville, N.Z., and Soifer, B.T. 1987, Ap.J. (Letters), 312, 235

Scoville, N.Z., Matthews, K., Carico, D., and Sanders, D.B. 1988, Ap.J. (Letters) (submitted)

Scoville, N.Z. and Norman, C.A. 1988, Ap.J., 332, 163

Scoville, N.Z., Sanders, D.B., Sargent, A.I., Soifer, B.T., Scott, S.L., and Lo, K.Y. 1986, Ap. J. (Letters), 311, L47

Scoville, N.Z., Soifer, T., Neugebauer, G., Young, J.S., Matthews, K., and Yerka, J. 1985, Ap.J., 289, 129

Soifer, B.T., et al.. 1984, Ap. J., 283, L1

Young, J.S., Kenney, J., Lord, S.D., and Schloerb, F.P. 1984, Ap.J. (Letters), 287, L65 